\title{
Geometria Diferencial de Curvas de Interseção de Duas Superfícies Implícitas
}

O. ALÉSSIO ${ }^{1}$, Departamento de Pós-Graduação, Mestrado em Matemática e Estatística, UNINCOR- Universidade Vale do Rio Verde de Três Corações, Av. Castelo Branco, 82, CEP: 37.410-000, Três Corações, MG, Brasil.

Resumo. Apresenta-se uma técnica para encontrar as propriedades geométricas da curva de interseção de duas superfícies implícitas, usando o teorema da função implícita.

\section{Introdução}

A principal motivação deste artigo é o problema de interseção de duas superfícies. Do ponto de vista histórico o problema de interseção de superfícies (SSI) vem de muitos séculos atrás. As clássicas curvas conhecidas como cônicas (elipse, hipérbole, parábola) são obtidas através da interseção entre duas superfícies: um plano e um cone. Muitos problemas podem ser reduzidos em interseção de superfícies, como por exemplo determinar: a equação paramétrica de curvas dadas implicitamente por duas superfícies, o conjunto de nível de uma função vetorial, máximos e mínimos via Multiplicadores de Lagrange com duas restrições, limites de integração de integrais triplas, soluções de sistemas não-lineares, formas complexas via modelagem geométrica. Para a interseção de superfícies em geral, não se consegue obter a equação paramétrica da curva de interseção, o que se faz é obter pontos ao longo da curva de interseção e para isso o método mais utilizado é o de caminhada [1]. O método de caminhada consiste em partir de um ponto inicial na curva de interseção e determinar iterativamente o próximo ponto até traçar a curva completamente. Propriedades geométricas locais da curva de interseção são aplicadas para adaptativamente determinar os passos da caminhada [1,3].

O problema de interseção de superfícies pode ser dos tipos: paramétrica-paramétrica, implícita-implícita e paramétrica-implícita. Para calcular a curva de interseção com precisão e eficiência, aproximações de ordem superior são necessárias, isto é, precisa-se obter as propriedades geométricas locais da curva de interseção. Enquanto geometria diferencial de curvas paramétricas pode ser encontrada em livros clássicos (Struik, 1950; Willmore, 1959; do Carmo, 1976), há pouca literatura de geometria diferencial de curvas de interseção. Willmore em seu livro [2], descreve como obter o vetor tangente, vetor normal e o vetor binormal da curva de interseção de duas superfícies implícitas usando o operador denotado por $\triangle$. Recentemente,

\footnotetext{
${ }^{1}$ osmaralessio@yahoo.com.br
} 
Ye e Maekawa [4], forneceram as propriedades geométricas da curva de interseção para os três tipos, usando o vetor $\alpha^{\prime \prime}$ como combinação linear dos vetores normais e $\alpha^{\prime \prime \prime}$ como combinação do vetor tangente e os vetores normais. Diferentemente dos trabalhos anteriores, pretende-se fornecer as propriedades geométricas da curva de interseção de duas superfícies implícitas, usando o teorema da função implícita. Neste trabalho, o cálculo das propriedades geométricas resume-se a resolver sistemas lineares de 2 equações e 2 variáveis, independente da ordem da derivada, isto é, se quiséssemos calcular a derivada de ordem 5 , teríamos que resolver um sistema linear de 2 equações e 2 variáveis, enquanto que o método de Willmore elevaria a complexidade a cada aplicação do operador $\triangle$ e o método de Ye e Maekawa teria complexos coeficientes das combinações lineares dos vetores envolvidos.

\section{Geometria Diferencial de Curvas}

\section{Curvas Parametrizadas pelo comprimento de arco}

Seja $x=x(s), y=y(s), z=z(s)$, ou em forma de vetor $\alpha(s)=(x(s), y(s), z(s))$, a curva parametrizada pelo comprimento de arco $s$. Então temos $\alpha^{\prime}(s)=\mathbf{t}$ e $\alpha^{\prime \prime}(s)=\mathbf{k}=k \mathbf{n}$, onde $\mathbf{t}$ é o vetor tangente unitário e $\mathbf{k}$ o vetor curvatura. Temos que a curvatura é dada por $k=\sqrt{\mathbf{k} \cdot \mathbf{k}}=\sqrt{\alpha^{\prime \prime} \cdot \alpha^{\prime \prime}}$. A terceira derivada $\alpha^{\prime \prime \prime}(s)$ é obtida por diferenciar $\alpha^{\prime \prime}(s)$ em relação a $s$, isto é, $\alpha^{\prime \prime \prime}(s)=k^{\prime} \mathbf{n}+k \mathbf{n}^{\prime}$, onde pode-se trocar $\mathbf{n}^{\prime}$ pela segunda equação do Triedro de Frenet, produzindo $\alpha^{\prime \prime \prime}(s)=-k^{2} \mathbf{t}+k^{\prime} \mathbf{n}+k \tau \mathbf{b}$. Como os vetores $\mathbf{t}, \mathbf{n}$ e $\mathbf{b}$ formam a base ortonormal com a orientação da mão-direita, a torção pode ser obtida por $\tau=\frac{\mathbf{b} \cdot \alpha^{\prime \prime \prime}(\mathbf{s})}{k}$.

\section{Superfícies Implícitas e Curvas Implícitas}

Definição 3.1 (Superfície Implícita). Seja $f: D \subset \mathbb{R}^{3} \longrightarrow \mathbb{R}$ uma aplicação diferenciável no aberto $D$. Dado $k \in \mathbb{R}$, lembramos que o conjunto de nivel $k$ de $f$ é o conjunto definido por $f^{-1}(k)=\{(x, y, z) \in D ; f(x, y, z)=k)$, isto é, $f^{-1}(k)$ é o conjunto solução em $D$ da equação $f(x, y, z)=k$. Formalmente:

$$
S=f^{-1}(0)=\left\{(x, y, z) \in \mathbb{R}^{3} \mid f(x, y, z)=0\right\} .
$$

Teorema 3.1 (Teor. da Função Implícita). Sejam $A \subset \mathbb{R}^{2} \times \mathbb{R}$ um aberto, $f$ : $A \rightarrow \mathbb{R}$ de classe $C^{k}, k \geq 1, f\left(x_{0}, y_{0}, z_{0}\right)=0$ e $\frac{\partial f}{\partial z}\left(x_{0}, y_{0}, z_{0}\right) \neq 0$. Então existem abertos $U \subset \mathbb{R}^{2}, V \subset \mathbb{R}$ com $\left(x_{0}, y_{0}, z_{0}\right) \in U \times V \subset A \subset \mathbb{R}^{2} \times \mathbb{R}$, tais que, para todo $(x, y) \in U$, existe um único $z=z(x, y) \in V$ tal que $f(x, y, z(x, y))=0$ e $z=z(x, y) \in C^{k}$.

Teorema 3.2. Sejam $f, g: A \subset \mathbb{R}^{3} \rightarrow \mathbb{R}$ funções de classe $C^{k}, k \geq 1$, no aberto $A$, $p_{0}=\left(x_{0}, y_{0}, z_{0}\right) \in A$, e $f\left(p_{0}\right)=c_{1}$ e $g\left(p_{0}\right)=c_{2}$. Se $\frac{\partial(f, g)}{\partial(y, z)}\left(p_{0}\right) \neq 0$ então existem abertos $U$ e $V$ tais que $\left(x_{0}, y_{0}, z_{0}\right) \in U \times V \subset A \subset \mathbb{R} \times \mathbb{R}^{2}$ e tais que, para todo $x \in U$, existe um único $(y, z)=(y(x), z(x)) \in V$ tal que $\left\{\begin{array}{l}f_{1}(x, y(x), z(x))=c_{1} \\ f_{2}(x, y(x), z(x))=c_{2} .\end{array}\right.$ 


\subsection{Representação Implícita das Curvas}

A representação implícita de uma curva espacial pode ser expressa como uma curva de interseção entre superfícies implícitas $f=0 \cap g=0$. De fato, é conhecido pelo teorema da função implícita que as equações $f=0$ e $g=0$, podem ser resolvidas para duas das variáveis em função da terceira. Por exemplo, se $\frac{\partial(f, g)}{\partial(y, z)}\left(P_{0}\right) \neq 0$, para alguma vizinhança de $x_{0}$ podemos resolver $f=0 \cap g=0$ para $y$ e $z$ como função de $x$, obtendo uma representação da seguinte forma $x=x, y=y(x), z=z(x)$ com $x$ sendo o parâmetro. Isto define localmente uma curva paramétrica $\alpha(x)=(x, y(x), z(x))$, que é solução de $f=0 \cap g=0$.

Sejam $f(x, y, z)=0$ e $g(x, y, z)=0$ superfícies implícitas. Vamos assumir que estas superfícies são todas regulares. Em outras palavras $\nabla f \neq 0, \nabla g \neq 0$. $\mathrm{O}$ vetor normal unitário da superfície implícita $f$ é dado por $N^{f}=\frac{\nabla f}{\|\nabla f\|}$.

\section{Trabalhos Existentes}

Com base nas propriedades geométricas das duas superfícies regulares, $f(x, y, z)$ e $g(x, y, z)$ que se intersectam, foram propostas técnicas para estimar ou determinar exatamente as propriedades locais da curva de interseção. As propriedades geométricas serão calculadas somente nos pontos cuja a interseção das superfícies seja transversal, isto é, os vetores normais de ambas as superfícies não são paralelos. Para o caso tangencial veja Ye e Maekawa [4].

Para ilustrar melhor os resultados que virão a seguir, veja a Figura 1

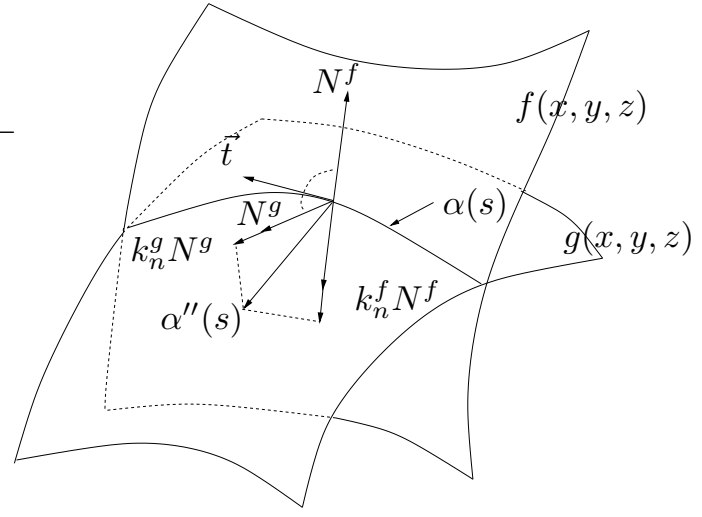

Figura 1: Interseção Transversal

\subsection{Vetor Tangente}

Barnhill e Kersey [1], quando se trata de uma interseção transversal, a forma mais usual para obter o vetor tangente em cada ponto $P$ é dada pelo produto vetorial dos vetores normais de ambas superfícies:

$$
\mathbf{t}=\frac{\mathbf{N}^{f}(u, v) \times \mathbf{N}^{g}(p, q)}{\left\|\mathbf{N}^{f}(u, v) \times \mathbf{N}^{g}(p, q)\right\|} .
$$




\subsection{Vetor Curvatura e Curvatura}

Ye e Maekawa [4] obtiveram expressões para a curvatura tanto nos pontos regulares quanto nos pontos singulares.

Para interseções transversais, o vetor curvatura da curva interseção no ponto $P$ é perpendicular ao vetor tangente, logo ele está no plano formado pelos vetores normais das duas superfícies. Assim, ele pode ser expresso como uma combinação linear dos dois vetores:

$$
\alpha^{\prime \prime}(s)=\alpha \mathbf{N}^{f}+\beta \mathbf{N}^{g},
$$

onde $\alpha$ e $\beta$ são as incógnitas. A curvatura normal em $P$ na direção $t$ é a projeção do vetor $\alpha^{\prime \prime}(s)$ sobre o vetor normal unitário $N$ da superfície em $P$ dado por

$$
k_{n}=\alpha^{\prime \prime}(s) \cdot \mathbf{N}=\kappa \mathbf{n} \cdot \mathbf{N} .
$$

Com isso, temos

$$
\begin{aligned}
& k_{n}^{f}=\alpha+\beta \cos (\theta) \\
& k_{n}^{g}=\alpha \cos (\theta)+\beta,
\end{aligned}
$$

onde $\theta$ é o ângulo entre os vetores normais $\mathbf{N}^{f}$ e $\mathbf{N}^{g}$.

Solucionando os coeficientes $\alpha$ e $\beta$ pelo sistema Eq.(4.4), e substituindo-os na Eq.(4.2), temos

$$
\alpha^{\prime \prime}=\frac{k_{n}^{f}-k_{n}^{g} \cos \theta}{\sin ^{2} \theta} \mathbf{N}^{f}+\frac{k_{n}^{g}-k_{n}^{f} \cos \theta}{\sin ^{2} \theta} \mathbf{N}^{g},
$$

onde $\cos \theta=\mathbf{N}^{f} \cdot \mathbf{N}^{g}$

A curvatura normal $k_{n}$ para a superfície implícita é obtida por usar $\frac{d^{2} f}{d s^{2}}$. A projeção do vetor $\frac{d^{2} f}{d s^{2}}$ sobre o vetor normal $N=\frac{\nabla f}{|\nabla f|}$ da superfície, é dado por:

$$
k_{n}^{f}=-\frac{f_{x x}\left(x^{\prime}\right)^{2}+f_{y y}\left(y^{\prime}\right)^{2}+f_{z z}\left(z^{\prime}\right)^{2}+2\left(f_{x y} x^{\prime} y^{\prime}+f_{y z} y^{\prime} z^{\prime}+f_{x z} x^{\prime} z^{\prime}\right)}{\sqrt[2]{\left(f_{x}\right)^{2}+\left(f_{y}\right)^{2}+\left(f_{z}\right)^{2}}},
$$

onde $x^{\prime}, y^{\prime}$ e $z^{\prime}$ são as coordenadas do vetor tangente $t=\alpha^{\prime}(s)$ dado pela Eq.(4.1).

A expressão da curvatura é dada exatamente por

$$
\kappa=\frac{1}{|\sin \theta|} \sqrt{\left(k_{n}^{f}\right)^{2}+\left(k_{n}^{g}\right)^{2}-2 k_{n}^{f} k_{n}^{g} \cos \theta} \quad \text { ou } \quad \kappa=\left\|\alpha^{\prime \prime}(s)\right\| .
$$

Willmore [2] descreve como obter a curvatura da curva de interseção para duas superfícies implícitas, considerando a curva de interseção representada pela equação $\alpha=\alpha(s)$, e sejam duas superfícies implícitas dadas por $f(\alpha(s))=0$ e $g(\alpha(s))=0$.

Como vetor tangente unitário da curva de interseção é ortogonal aos vetores normais de ambas as superfícies. Assim, se $\nabla f=\left(\frac{\partial f}{\partial x}, \frac{\partial f}{\partial y}, \frac{\partial f}{\partial z}\right)$, segue que $t=\alpha^{\prime}(s)$ é paralelo a

$$
\nabla f \times \nabla g=h .
$$


Diz-se que $\lambda \alpha^{\prime}=\nabla f \times \nabla g$. Então $\lambda x^{\prime}=h_{1}, \lambda y^{\prime}=h_{2}, \lambda z^{\prime}=h_{3} \mathrm{e}$

$$
\lambda \frac{d}{d s}=\left(h_{1} \frac{\partial}{\partial x}+h_{2} \frac{\partial}{\partial y}+h_{3} \frac{\partial}{\partial z}\right) .
$$

É conveniente denotar o operador( 4.8) por $\Delta$. Portanto

$$
\Delta \alpha=\mathbf{h} .
$$

Da definição de $\lambda$ e $h$ segue que

$$
\lambda \mathbf{t}=\mathbf{h},
$$

e assim

$$
\lambda^{2}=\mathbf{h}^{2} .
$$

Operando em (4.10) com $\Delta$ tem-se

$$
\lambda^{2} k \mathbf{n}+\lambda \lambda^{\prime} \mathbf{t}=\Delta \mathbf{h} .
$$

Aplicando o produto vetorial de (4.9) e (4.12) temos:

$$
\lambda^{3} k \mathbf{b}=\mathbf{h} \times \Delta \mathbf{h}=\mathbf{k},
$$

logo a curvatura da curva é

$$
k=\frac{\|\mathbf{k}\|}{\lambda^{3}} .
$$

O vetor curvatura $\alpha^{\prime \prime}(s)$ é dado por

$$
\alpha^{\prime \prime}(s)=\frac{\mathbf{k}}{\lambda^{3}} \times \mathbf{t}=k \mathbf{b} \times \mathbf{t} .
$$

\subsection{Torção}

Ye e Maekawa [4] obtiveram também expressões da torção para interseções transversais.

Como $\mathbf{N}^{f}$ e $\mathbf{N}^{g}$ estão no plano normal (plano gerado por $\mathbf{n}$ e b), os termos $k^{\prime} \mathbf{n}+k \tau \mathbf{b}$ de $\alpha^{\prime \prime \prime}(s)=-k^{2} \mathbf{t}+k^{\prime} \mathbf{n}+k \tau \mathbf{b}$ pode ser trocado pela combinação linear $\gamma \mathbf{N}^{f}+\delta \mathbf{N}^{g}$. Assim temos

$$
\alpha^{\prime \prime \prime}(s)=-\kappa^{2} t+\gamma \mathbf{N}^{f}+\delta \mathbf{N}^{g} .
$$

Agora, se projetarmos $\alpha^{\prime \prime \prime}(s)$ sobre os vetores normais unitários $N^{f}$ e $N^{g}$ em $P$ e denotarmos por $\lambda_{n}^{f}$ e $\lambda_{n}^{g}$, respectivamente, temos

$$
\begin{aligned}
& \lambda_{n}^{f}=\gamma+\delta \cos (\theta) \\
& \lambda_{n}^{g}=\gamma \cos (\theta)+\delta .
\end{aligned}
$$

Resolvendo o sistema linear (4.17) para os escalares $\gamma, \delta$ e substituindo em (4.16) tem-se

$$
\alpha^{\prime \prime \prime}=-\kappa^{2} \mathbf{t}+\frac{\lambda_{n}^{f}-\lambda_{n}^{g} \cos \theta}{\sin ^{2} \theta} \mathbf{N}^{f}+\frac{\lambda_{n}^{g}-\lambda_{n}^{f} \cos \theta}{\sin ^{2} \theta} \mathbf{N}^{g} .
$$


Os parâmetros $\lambda_{n}^{f}$ e $\lambda_{n}^{g}$ para ambas as superfícies implícitas são obtidos usando $\frac{d^{3} f}{d s^{3}}$. A projeção do $\frac{d^{3} f}{d s^{3}}$ sobre o vetor normal $N=\frac{\nabla f}{|\nabla f|}$ da superfície, é dado por:

$$
\lambda_{n}=\frac{f_{x} x^{\prime \prime \prime}+f_{y} y^{\prime \prime \prime}+f_{z} z^{\prime \prime \prime}}{\sqrt[2]{\left(f_{x}\right)^{2}+\left(f_{y}\right)^{2}+\left(f_{z}\right)^{2}}}=-\frac{F_{1}+F_{2}+F_{3}}{\sqrt[2]{\left(f_{x}\right)^{2}+\left(f_{y}\right)^{2}+\left(f_{z}\right)^{2}}},
$$

onde

$$
\begin{aligned}
& F_{1}=f_{x x x}\left(x^{\prime}\right)^{3}+f_{y y y}\left(y^{\prime}\right)^{3}+f_{z z z}\left(z^{\prime}\right)^{3}, \\
& F_{2}=3\left[f_{x x y}\left(x^{\prime}\right)^{2} y^{\prime}+f_{x x z}\left(x^{\prime}\right)^{2} z^{\prime}+f_{x y y} x^{\prime}\left(y^{\prime}\right)^{2}+f_{y y z}\left(y^{\prime}\right)^{2} z^{\prime}+f_{x z z} x^{\prime}\left(z^{\prime}\right)^{2}+\right. \\
& \left.\quad f_{y z z} y^{\prime}\left(z^{\prime}\right)^{2}+f_{x y z} x^{\prime} y^{\prime} z^{\prime}\right], \\
& F_{3}=3\left[f_{x x} x^{\prime} x^{\prime \prime}+f_{y y} y^{\prime} y^{\prime \prime}+f_{z z} z^{\prime} z^{\prime \prime}+f_{x y}\left(x^{\prime \prime} y^{\prime}+x^{\prime} y^{\prime \prime}\right)+f_{y z}\left(y^{\prime \prime} z^{\prime}+y^{\prime} z^{\prime \prime}\right)+\right. \\
& \left.\quad f_{x z}\left(x^{\prime \prime} z^{\prime}+x^{\prime} z^{\prime \prime}\right)\right]
\end{aligned}
$$

onde $x^{\prime}, y^{\prime}$ e $z^{\prime}$ são dados pela Eq.(4.1) e $x^{\prime \prime}, y^{\prime \prime}$ e $z^{\prime \prime}$ são dados pela Eq.(4.5).

Finalmente, a torção pode ser obtida de $\alpha^{\prime \prime \prime}(s)=-k^{2} \mathbf{t}+k^{\prime} \mathbf{n}+k \tau \mathbf{b}$ por

$$
\tau=\frac{\mathbf{b} \cdot \alpha^{\prime \prime \prime}}{k},
$$

onde $\kappa$ é a curvatura e o vetor binormal pela expressão $\mathbf{b}=\mathbf{t} \times \mathbf{n}$.

Willmore [2] descreve como obter a torção da curva de interseção para duas superfícies implícitas.

Aplicando o operador $\Delta$ na Eq.(4.13) temos

$$
\lambda\left(\lambda^{3} k\right)^{\prime} \mathbf{b}-\lambda^{4} k \tau \mathbf{n}=\Delta \mathbf{k} .
$$

Fazendo o produto escalar de (4.12) e de (4.21) temos

$$
-\lambda^{6} k^{2} \tau=\Delta \mathbf{h} \cdot \Delta \mathbf{k},
$$

logo a torção é

$$
\tau=-\frac{\Delta \mathbf{h} \cdot \Delta \mathbf{k}}{\lambda^{6} k^{2}}
$$

\section{Método usando o Teorema da Função Implícita}

\subsection{Vetor Tangente}

Sem perda de generalidade, podemos assumir que a matriz jacobiana $\frac{\partial(f, g)}{\partial(y, z)}\left(P_{0}\right) \neq 0$, então temos localmente uma curva paramétrica $\alpha(x)=(x, y(x), z(x))$. Portanto temos que o ponto da curva $P_{0}=\alpha\left(x_{0}\right)$ satisfaz as duas relações da forma

$$
\left\{\begin{array}{l}
f\left(x_{0}, y\left(x_{0}\right), z\left(x_{0}\right)\right)=0 \\
g\left(x_{0}, y\left(x_{0}\right), z\left(x_{0}\right)\right)=0
\end{array} .\right.
$$


Derivando as equações em relação a $x$, temos

$$
\left\{\begin{array} { l } 
{ \frac { \partial f } { \partial x } + \frac { \partial f } { \partial y } \dot { y } + \frac { \partial f } { \partial z } \dot { z } = 0 } \\
{ \frac { \partial g } { \partial x } + \frac { \partial g } { \partial y } \dot { y } + \frac { \partial g } { \partial z } \dot { z } = 0 }
\end{array} \Longleftrightarrow \left\{\begin{array}{l}
\frac{\partial f}{\partial y} \dot{y}+\frac{\partial f}{\partial z} \dot{z}=-\frac{\partial f}{\partial x} \\
\frac{\partial g}{\partial y} \dot{y}+\frac{\partial g}{\partial z} \dot{z}=-\frac{\partial g}{\partial x}
\end{array}\right.\right.
$$

onde $\dot{y}=\frac{d y}{d x}$ e $\dot{z}=\frac{d z}{d x}$. O vetor tangente da curva $\alpha(x)$ no ponto $\alpha\left(x_{0}\right)=P_{0}$ é dado por $\dot{\alpha}\left(x_{0}\right)=\left(1, \dot{y}\left(x_{0}\right), \dot{z}\left(x_{0}\right)\right)$. Para obter este vetor tangente, devemos resolver o sistema $(5.2)$.

\subsection{Vetor Curvatura e Curvatura}

Derivando as equações dadas no sistema (5.2) em relação a $x$, temos

$$
\left\{\begin{array}{l}
\frac{\partial f}{\partial y} \ddot{y}+\frac{\partial f}{\partial z} \ddot{z}=-\frac{\partial^{2} f}{\partial x \partial x}-\frac{\partial^{2} f}{\partial y \partial y} \dot{y} \dot{y}-\frac{\partial^{2} f}{\partial z \partial z} \dot{z} \dot{z}-2 \frac{\partial^{2} f}{\partial x \partial y} \dot{y}-2 \frac{\partial^{2} f}{\partial x \partial z} \dot{z}-2 \frac{\partial^{2} f}{\partial y \partial z} \dot{y} \dot{z} \\
\frac{\partial g}{\partial y} \ddot{y}+\frac{\partial g}{\partial z} \ddot{z}=-\frac{\partial^{2} g}{\partial x \partial x}-\frac{\partial^{2} g}{\partial y \partial y} \dot{y} \dot{y}-\frac{\partial^{2} g}{\partial z \partial z} \dot{z} \dot{z}-2 \frac{\partial^{2} g}{\partial x \partial y} \dot{y}-2 \frac{\partial^{2} g}{\partial x \partial z} \dot{z}-2 \frac{\partial^{2} g}{\partial y \partial z} \dot{y} \dot{z}
\end{array}\right.
$$

onde $\ddot{y}=\frac{d^{2} y}{d x^{2}}$ e $\ddot{z}=\frac{d^{2} z}{d x^{2}}$. O vetor derivada segunda da curva $\alpha(x)$ no ponto $\boldsymbol{\alpha}\left(x_{0}\right)=P_{0}$ é dado por $\ddot{\alpha}\left(x_{0}\right)=\left(0, \ddot{y}\left(x_{0}\right), \ddot{z}\left(x_{0}\right)\right)$. A curvatura da curva no ponto $\boldsymbol{\alpha}\left(x_{0}\right)=P_{0}$ é $\kappa\left(x_{0}\right)=\frac{\left\|\dot{\alpha}\left(x_{0}\right) \times \ddot{\alpha}\left(x_{0}\right)\right\|}{\left\|\dot{\alpha}\left(x_{0}\right)\right\|^{3}}$. O vetor curvatura é $\alpha^{\prime \prime}\left(x_{0}\right)=k \mathbf{n}\left(x_{0}\right)$, onde $\mathbf{n}\left(x_{0}\right)=\mathbf{b}\left(x_{0}\right) \times \mathbf{t}\left(x_{0}\right)$ e $\mathbf{b}\left(x_{0}\right)=\frac{\dot{\alpha}\left(x_{0}\right) \times \ddot{\alpha}\left(x_{0}\right)}{\left\|\dot{\alpha}\left(x_{0}\right) \times \ddot{\alpha}\left(x_{0}\right)\right\|}$.

\subsection{Torção}

Derivando as equações dadas no sistema (5.3) em relação a $x$, temos

$$
\left\{\begin{aligned}
\frac{\partial f}{\partial y} \dddot{y}+\frac{\partial f}{\partial z} \dddot{z}= & -\frac{\partial^{3} f}{\partial x \partial x \partial x}-\frac{\partial^{3} f}{\partial y \partial y \partial y}(\dot{y})^{3}-\frac{\partial^{3} f}{\partial z \partial z \partial z}(\dot{z})^{3}-3 \frac{\partial^{3} f}{\partial x \partial x \partial y} \dot{y}-3 \frac{\partial^{3} f}{\partial x \partial x \partial z} \dot{z} \\
& -3 \frac{\partial^{3} f}{\partial x \partial y \partial y}(\dot{y})^{2}-3 \frac{\partial^{3} f}{\partial y \partial y \partial z}(\dot{y})^{2} \dot{z}-3 \frac{\partial^{3} f}{\partial x \partial z \partial z}(\dot{z})^{2}-3 \frac{\partial^{3} f}{\partial y \partial z \partial z} \dot{y}(\dot{z})^{2} \\
& -6 \frac{\partial^{3} f}{\partial x \partial y \partial z} \dot{y} \dot{z}-3 \frac{\partial^{2} f}{\partial y \partial y} \dot{y} \ddot{y}-3 \frac{\partial^{2} f}{\partial z \partial z} \dot{z} \ddot{z}-3 \frac{\partial^{2} f}{\partial x \partial y} \ddot{y}-3 \frac{\partial^{2} f}{\partial y \partial z}(\ddot{y} \dot{z}+\dot{y} \ddot{z}) \\
& -3 \frac{\partial f}{\partial x \partial z} \ddot{z} \\
\frac{\partial g}{\partial y} \dddot{y}+\frac{\partial g}{\partial z} \dddot{z}= & -\frac{\partial^{3} g}{\partial x \partial x \partial x}-\frac{\partial^{3} g}{\partial y \partial y \partial y}(\dot{y})^{3}-\frac{\partial^{3} g}{\partial z \partial z \partial z}(\dot{z})^{3}-3 \frac{\partial^{3} g}{\partial x \partial x \partial y} \dot{y}-3 \frac{\partial^{3} g}{\partial x \partial x \partial z} \dot{z} \\
& -3 \frac{\partial^{3} g}{\partial x \partial y \partial y}(\dot{y})^{2}-3 \frac{\partial^{3} g}{\partial y \partial y \partial z}(\dot{y})^{2} \dot{z}-3 \frac{\partial^{3} g}{\partial x \partial z \partial z}(\dot{z})^{2}-3 \frac{\partial^{3} g}{\partial y \partial z \partial z} \dot{y}(\dot{z})^{2} \\
& -6 \frac{\partial^{3} g}{\partial x \partial y \partial z} \dot{y} \dot{z}-3 \frac{\partial^{2} g}{\partial y \partial y} \dot{y} \ddot{y}-3 \frac{\partial^{2} g}{\partial z \partial z} \dot{z} \ddot{z}-3 \frac{\partial^{2} g}{\partial x \partial y} \ddot{y}-3 \frac{\partial^{2} g}{\partial y \partial z}(\ddot{y} \dot{z}+\dot{y} \ddot{z}) \\
& -3 \frac{\partial g}{\partial x \partial z} \ddot{z}
\end{aligned}\right.
$$

onde $\dddot{y}=\frac{d^{3} y}{d x^{3}}$ e $\dddot{z}=\frac{d^{3} z}{d x^{3}}$. O vetor $\dddot{\alpha}(x)$ no ponto $\alpha\left(x_{0}\right)=P_{0}$ é dado por $\dddot{\alpha}\left(x_{0}\right)=\left(0, \ddot{y}\left(x_{0}\right), \dddot{z}\left(x_{0}\right)\right)$. A torção da curva no ponto $\boldsymbol{\alpha}\left(x_{0}\right)=P_{0}$ é

$$
\tau\left(x_{0}\right)=\frac{\left(\dot{\alpha}\left(x_{0}\right) \times \ddot{\alpha}\left(x_{0}\right)\right) \cdot \dddot{\alpha}\left(x_{0}\right)}{\left\|\dot{\alpha}\left(x_{0}\right) \times \ddot{\alpha}\left(x_{0}\right)\right\|^{2}} .
$$

\section{Exemplo}

Será calculado o vetor tangente, vetor curvatura, curvatura e a torção no ponto $P\left(\frac{1}{2}, \frac{1}{2}, \frac{\sqrt{2}}{2}\right)$ da curva de interseção da esfera de raio 1 com o cilindro deslocado em 
$\frac{1}{2}$ no eixo $x$ e raio $\frac{1}{2}$, cujas equações implícitas são dadas respectivamente por:

$$
\left\{\begin{array}{l}
f(x, y, z)=x^{2}+y^{2}+z^{2}-1=0 \\
g(x, y, z)=x^{2}+y^{2}-x=0
\end{array} .\right.
$$
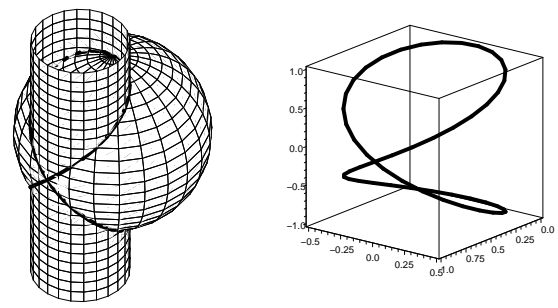

Figura 2: Esfera X Cilindro e a Curva de Interseção

\subsection{Método Ye e Maekawa}

Por Barnhill e Kersey [1] o vetor tangente é dado por $\mathbf{t}=\frac{N^{f}(u, v) \times N^{g}(p, q)}{\left|N^{f}(u, v) \times N^{g}(p, q)\right|}$.

No ponto $P\left(\frac{1}{2}, \frac{1}{2}, \frac{\sqrt{2}}{2}\right)$ temos $\mathbf{N}^{f}=\left(\frac{1}{2}, \frac{1}{2}, \frac{\sqrt{2}}{2}\right)$ e $\mathbf{N}^{g}=(0,1,0)$. Portanto o vetor tangente é $\mathbf{t}\left(s_{0}\right)=\left(-\frac{\sqrt{6}}{3}, 0, \frac{\sqrt{3}}{3}\right)$. O vetor curvatura é dado pela Eq.(4.5) $\alpha^{\prime \prime}(s)=\frac{k_{n}^{f}-k_{n}^{g} \cos \theta}{\sin ^{2} \theta} \mathbf{N}^{f}+\frac{k_{n}^{g}-k_{n}^{f} \cos \theta}{\sin ^{2} \theta} \mathbf{N}^{g}$, e a expressão da curvatura é dada exatamente por $\kappa=\frac{1}{|\sin \theta|} \sqrt{\left(k_{n}^{f}\right)^{2}+\left(k_{n}^{g}\right)^{2}-2 k_{n}^{f} k_{n}^{g} \cos \theta}$.

Agora precisamos calcular as duas curvaturas normais $k_{n}^{f}$ e $k_{n}^{g}$ e os ângulos $\cos \theta$ e $\sin \theta$ em $P$. Para calcular $k_{n}^{f}$ e $k_{n}^{g}$ usamos a Eq.(4.6). No ponto $P\left(\frac{1}{2}, \frac{1}{2}, \frac{\sqrt{2}}{2}\right)$ temos: $k_{n}^{f}=-1, k_{n}^{g}=-\frac{4}{3}, \cos \theta=\frac{1}{2}$ e $\sin \theta=\frac{\sqrt{3}}{2}$. Portanto o vetor curvatura é $\alpha^{\prime \prime}\left(s_{0}\right)=\left(-\frac{2}{9},-\frac{4}{3},-\frac{2 \sqrt{2}}{9}\right)$ e a curvatura é $\kappa=\frac{2}{3} \sqrt{\frac{13}{3}}$.

$\mathrm{O}$ vetor $\alpha^{\prime \prime \prime}$ é dado pela Eq.(4.18) $\alpha^{\prime \prime \prime}=-\kappa^{2} \mathbf{t}+\frac{\lambda_{n}^{f}-\lambda_{n}^{g} \cos \theta}{\sin ^{2} \theta} \mathbf{N}^{f}+\frac{\lambda_{n}^{g}-\lambda_{n}^{f} \cos \theta}{\sin ^{2} \theta} \mathbf{N}^{g}$ e a expressão da torção é dada por $\tau=\frac{\mathbf{b} \cdot \alpha^{\prime \prime \prime}}{k}$. Os parâmetros $\lambda_{n}^{f}$ e $\lambda_{n}^{g}$ são dados pela Eq.(4.19). No ponto $P\left(\frac{1}{2}, \frac{1}{2}, \frac{\sqrt{2}}{2}\right)$ temos: $\lambda_{n}^{f}=0, \lambda_{n}^{g}=\frac{4 \sqrt{6}}{9}$.

Portanto $\alpha^{\prime \prime \prime}\left(s_{0}\right)=\left(\frac{40 \sqrt{6}}{81}, \frac{4 \sqrt{6}}{9},-\frac{76 \sqrt{3}}{81}\right)$. Para calcular a torção precisamos encontrar o vetor binormal $\mathbf{b}$, mas antes devemos encontrar o vetor $\mathbf{n}$, isto é,

$\mathbf{n}=\frac{\mathbf{k}}{k}=\left(-\frac{1}{3} \sqrt{\frac{3}{13}},-2 \sqrt{\frac{3}{13}},-\frac{1}{3} \sqrt{\frac{6}{13}}\right)$ e $\mathbf{b}=\mathbf{t} \times \mathbf{n}=\left(\frac{2 \sqrt{13}}{13},-\frac{\sqrt{13}}{13}, \frac{2 \sqrt{26}}{13}\right)$. Então a torção é $\tau=\frac{\mathbf{b} \cdot \alpha^{\prime \prime \prime}}{k}=\frac{6 \sqrt{2}}{13}$.

\subsection{Método Willmore}

Agora o vetor tangente unitário é calculado usando as Eqs.(4.7),(4.10) e (4.11). 
A Eq.(4.7) é dada por $h=\nabla f \times \nabla g=(-4 y z, 4 x z-2 z, 2 y)$, pois $\nabla f=$ $(2 x, 2 y, 2 z)$ e $\nabla g=(2 x-1,2 y, 0)$. No ponto $P\left(\frac{1}{2}, \frac{1}{2}, \frac{\sqrt{2}}{2}\right)$, temos $\nabla f=(1,1, \sqrt{2})$ e $\nabla g=(0,1,0)$. Portanto $h\left(P_{0}\right)-=(-\sqrt{2}, 0,1)$. Da Eq.(4.10) temos $\lambda t=(-\sqrt{2}, 0,1)$, consequentemente $\lambda x^{\prime}=-\sqrt{2}, \lambda y^{\prime}=0, \lambda z^{\prime}=1$. Da Eq.(4.11) tem-se $\lambda^{2}=\mathbf{h}^{2}=$ $3 \Longrightarrow \lambda= \pm \sqrt{3}$. Obtendo o vetor tangente $\mathbf{t}= \pm\left(-\frac{\sqrt{6}}{3}, 0, \frac{\sqrt{3}}{3}\right)$.

Escolhendo $\mathbf{t}=\left(-\frac{\sqrt{6}}{3}, 0, \frac{\sqrt{3}}{3}\right) \Rightarrow \lambda=\sqrt{3}$. Usando o operador $\Delta$ em $h$ temos: $\Delta h=\left(\left(h_{1} \frac{\partial}{\partial x}+h_{2} \frac{\partial}{\partial y}+h_{3} \frac{\partial}{\partial z}\right)(-4 y z),\left(h_{1} \frac{\partial}{\partial x}+h_{2} \frac{\partial}{\partial y}+h_{3} \frac{\partial}{\partial z}\right)(4 x z-2 z),\left(h_{1} \frac{\partial}{\partial x}+h_{2} \frac{\partial}{\partial y}+\right.\right.$ $\left.\left.h_{3} \frac{\partial}{\partial z}\right)(2 y)\right)=\left(-4 z h_{2}-4 y h_{3}, 4 z h_{1}+(4 x-2) h_{3}, 2 h_{2}\right)$, como $\lambda x^{\prime}=h_{1}, \lambda y^{\prime}=h_{2}, \lambda z^{\prime}=$ $h_{3}$, tem-se $\Delta h=\lambda\left(-4 y^{\prime} z-4 y z^{\prime}, 4 x^{\prime} z+4 x z^{\prime}-2 z^{\prime}, 2 y^{\prime}\right)$. Usando as coordenadas do ponto $P\left(\frac{1}{2}, \frac{1}{2}, \frac{\sqrt{2}}{2}\right)$ e do vetor tangente, $\Delta \mathbf{h}\left(P_{0}\right)=(-2,-4,0)$. Aplicando o produto vetorial de $\mathbf{h}\left(P_{0}\right)$ e $\Delta \mathbf{h}\left(P_{0}\right)$ temos: $\mathbf{k}=\mathbf{h}\left(P_{0}\right) \times \Delta \mathbf{h}\left(P_{0}\right)=(4,-2,4 \sqrt{2})$, então a curvatura da curva é $k=\frac{\|\mathbf{k}\|}{\lambda^{3}}=\frac{2 \sqrt{13}}{3 \sqrt{3}}=\frac{2}{3} \sqrt{\frac{13}{3}}$. O vetor curvatura $\alpha^{\prime \prime}(s)$ é dado pela Eq.(4.15) $\alpha^{\prime \prime}(s)=\left(-\frac{2}{9},-\frac{4}{3},-\frac{2 \sqrt{2}}{9}\right)$.

Fazendo o produto vetorial de $\mathbf{h}$ por $\Delta \mathbf{h}$ temos $\mathbf{k}=\mathbf{h} \times \Delta \mathbf{h}=(-4 \mathrm{yz}, 4 \mathrm{xz}-2 \mathrm{z}, 2 \mathrm{y}) \times$ $\lambda\left(-4 y^{\prime} z-4 y z^{\prime}, 4 x^{\prime} z+4 x z^{\prime}-2 z^{\prime}, 2 y^{\prime}\right)$

Usando o operador $\Delta$ em $\mathbf{k}$ temos: $\Delta \mathbf{k}=\mathbf{h} \times \Delta^{2} \mathbf{h}=(-4 y z, 4 x z-2 z, 2 y) \times$ $4 \lambda^{2}\left(-y^{\prime \prime} z-2 y^{\prime} z^{\prime}-y z^{\prime \prime}, x^{\prime \prime} z+2 x^{\prime} z^{\prime}+x z^{\prime \prime}-\frac{1}{2} z^{\prime \prime}, \frac{1}{2} y^{\prime \prime}\right)$

Substituindo as coordenadas do ponto $P\left(\frac{1}{2}, \frac{1}{2}, \frac{\sqrt{2}}{2}\right)$, do vetor tangente, e do vetor curvatura, temos $\Delta \mathbf{k}=\left(\frac{28 \sqrt{2}}{3}, \frac{4 \sqrt{2}}{3}, \frac{56}{3}\right)$.

Pela Eq.(4.23), a torção é o produto escalar de $\Delta \mathbf{h}$ por $\Delta \mathbf{k}$ dividido por $\lambda^{6} k^{2}$, $\operatorname{logo}$ temos $\tau=-\frac{\Delta \mathbf{h} \cdot \Delta \mathbf{k}}{\lambda^{6} k^{2}}=\frac{6 \sqrt{2}}{13}$.

\subsection{Método Função Implícita}

Escolhendo $x$ como parâmetro, pois det $\left(\begin{array}{ll}\frac{\partial f}{\partial y} & \frac{\partial f}{\partial z} \\ \frac{\partial g}{\partial y} & \frac{\partial g}{\partial z}\end{array}\right) \neq 0$ no ponto $P\left(\frac{1}{2}, \frac{1}{2}, \frac{\sqrt{2}}{2}\right)$, o sistema (5.2) torna-se $\left\{\begin{array}{l}2 y \dot{y}+2 z \dot{z}=-2 x \\ 2 y \dot{y}=-2 x+1\end{array}\right.$. Substituindo $x=\frac{1}{2}, y=\frac{1}{2}$ e $z=\frac{\sqrt{2}}{2}$, $\operatorname{temos}\left\{\begin{array}{l}\dot{y}+\sqrt{2} \dot{z}=-1 \\ \dot{y}=-1+1\end{array} \Longleftrightarrow\left\{\begin{array}{l}\dot{z}=-\frac{\sqrt{2}}{2} \\ \dot{y}=0 .\end{array}\right.\right.$

$\mathrm{O}$ vetor $\dot{\alpha}$ é $\dot{\alpha}\left(\frac{1}{2}\right)=\left(1,0,-\frac{\sqrt{2}}{2}\right)$. O vetor tangente unitário na direção contrária de $\dot{\alpha}$ é $\mathbf{t}\left(\frac{1}{2}\right)=\left(-\frac{\sqrt{6}}{3}, 0, \frac{\sqrt{3}}{3}\right)$.

O sistema $(5.3)$ torna-se $\left\{\begin{array}{l}2 y \ddot{y}+2 z \ddot{z}=-2-2 \dot{y} \dot{y}-2 \dot{z} \dot{z} \\ 2 y \ddot{y}=-2-2 \dot{y} \dot{y} .\end{array} \quad\right.$ Substituindo $x=$ $\frac{1}{2}, y=\frac{1}{2}, z=\frac{\sqrt{2}}{2}, \dot{x}=1, \dot{y}=0, \dot{z}=-\frac{\sqrt{2}}{2}$, tem-se $\left\{\begin{array}{l}\ddot{z}=-\frac{\sqrt{2}}{2} \\ \ddot{y}=-2 .\end{array} \quad\right.$ Logo o vetor derivada segunda é $\ddot{\alpha}\left(\frac{1}{2}\right)=\left(0,-2,-\frac{\sqrt{2}}{2}\right)$.

A curvatura é $\kappa(u)=\frac{\|\dot{\alpha}(u) \times \ddot{\alpha}(u)\|}{\|\dot{\alpha}(u)\|^{3}}=\frac{2}{3} \sqrt{\frac{13}{3}}$. 
O vetor curvatura é $\alpha^{\prime \prime}\left(x_{0}\right)=k \mathbf{n}\left(x_{0}\right)$, onde $\mathbf{n}\left(x_{0}\right)=\mathbf{b}\left(x_{0}\right) \times \mathbf{t}\left(x_{0}\right)$ e $\mathbf{b}\left(x_{0}\right)=$ $\frac{\dot{\alpha}\left(x_{0}\right) \times \ddot{\alpha}\left(x_{0}\right)}{\left\|\dot{\alpha}\left(x_{0}\right) \times \ddot{\alpha}\left(x_{0}\right)\right\|}$.

$\operatorname{Temos} \mathbf{b}\left(x_{0}\right)=\left(-\frac{2 \sqrt{13}}{13}, \frac{\sqrt{13}}{13},-\frac{4 \sqrt{26}}{26}\right), \mathbf{n}\left(x_{0}\right)=\left(-\frac{\sqrt{39}}{39},-\frac{2 \sqrt{39}}{13},-\frac{\sqrt{78}}{39}\right) \mathrm{e}$ $\alpha^{\prime \prime}\left(x_{0}\right)=\frac{2}{3} \sqrt{\frac{13}{3}}\left(-\frac{\sqrt{39}}{39},-\frac{2 \sqrt{39}}{13},-\frac{\sqrt{78}}{39}\right)$.

O vetor curvatura é $\alpha^{\prime \prime}\left(x_{0}\right)=\left(-\frac{2}{9},-\frac{4}{3},-\frac{2 \sqrt{2}}{9}\right)$.

O sistema (??) torna-se $\left\{\begin{array}{l}2 y \dddot{y}+2 z \dddot{z}=-4 \dot{y} \ddot{y}-2 \dot{y} \ddot{y}-4 \dot{z} \ddot{z}-2 \dot{z} \ddot{z} \\ 2 y \dddot{y}=-4 \dot{y} \ddot{y}-2 \dot{y} \ddot{y}\end{array}\right.$. Substituindo $x=\frac{1}{2}, y=\frac{1}{2}, z=\frac{\sqrt{2}}{2}, \dot{x}=1, \dot{y}=0, \dot{z}=-\frac{\sqrt{2}}{2}, \ddot{x}=0, \ddot{y}=-2, \ddot{z}=-\frac{\sqrt{2}}{2}$, temos $\left\{\begin{array}{l}\dddot{y}+\sqrt{2} \dddot{z}=-2-1 \\ \dddot{y}=0\end{array} \Longleftrightarrow\left\{\begin{array}{l}\dddot{z}=-\frac{3 \sqrt{2}}{2} \\ \dddot{y}=0\end{array}\right.\right.$. Logo o vetor terceira derivada é $\dddot{\alpha}\left(\frac{1}{2}\right)=\left(0,0,-\frac{3 \sqrt{2}}{2}\right)$.

A torção é $\tau(u)=\frac{(\dot{\alpha}(u) \times \ddot{\alpha}(u)) \cdot \dddot{\alpha}}{\|\dot{\alpha}(u) \times \ddot{\alpha}(u)\|^{2}}=\frac{6 \sqrt{2}}{13}$.

Abstract. We present a method for computing the differential geometry properties of the intersection curve of two implicit surfaces, using the Implicit Function Theorem.

\section{Referências}

[1] R.E. Barnhill, S.N. Kersey, A marching method for parametric surface/surface intersection, Computer Aided Geometric Design, 7, No. 1-4, (1990), 257-280.

[2] T.J. Willmore, "An Introduction to Differential Geometry". Clarendon Press, Oxford, 1959.

[3] S.T. Wu, O. Alessio, S.I.R. Costa, On estimating local geometric properties of intersection curves. In "Proceedings of SIBGRAPI 2000", pp. 152-159, 2000.

[4] X. Ye, T. Maekawa, Differential geometry of intersection curves of two surfaces, Computer Aided Geometric Design, 16 (1999), 767-788. 